\title{
Una propuesta de mejora para el auto aprendizaje de la Competencia Comunicativa Oral en español: R/ele/CCO
}

\section{$M^{a}$ del Mar Sáinz González ${ }^{a}$, Francesca Romero Forteza ${ }^{b}$}

a'Universitat Politècnica de València (Spain, masaigon@upv.es), bUniversitat Politècnica de València (Spain, fromero@upv.es)

\begin{abstract}
This investigation focuses on the lack of web resources for Autonomous learning of oral communicative competence in Spanish, which we think can applied to other languages. The oral communicative competence is, according to the MCERL, the aim in and of itself when we learn a language. The Web resources are centered in the learning of languages based on exercises of trial and error and repetition, leaving aside the communicative level of the language. Therefore, we propose a web resource for an autonomous learning of Spanish as a foreign language which is focused on the learning of conversational characteristics in Spanish.
\end{abstract}

Keywords: Oral communicative competence, Autonomous learning of languages, ELE, Design Web based resources

\section{Resumen}

Este trabajo se centra en la falta de recursos web centrados en el auto aprendizaje de la competencia comunicativa oral en español, que pensamos, podemos hacer extensible a otras lenguas. La competencia comunicativa oral es, según el MCERL, el fin en si mismo cuando aprendemos una lengua. Los recursos web están centrados en el aprendizaje de lenguas basados en ejercicios de prueba y error y repetición, dejando de lado el plano comunicativo de la lengua. Por ende, proponemos un recurso web para el auto aprendizaje de español como lengua extranjera centrado en el aprendizaje de rasgos conversacionales en español.

Palabras clave: competencia comunicativa oral, auto aprendizaje de lenguas, ELE, diseño de recursos web.

\section{Introducción}

No hace demasiados años afirmábamos que las tecnologías de la información y la comunicación -TIC a partir de ahora- estaban invadiendo todas y cada una de las parcelas de nuestra vida. Pasado un tiempo constatamos que las TIC se han incorporado a nuestras vidas 
y han modificado nuestra cotidianidad. Estas herramientas han penetrado en todos los ámbitos sociales desde el profesional al personal. Cada vez realizamos más actividades a través de las TIC desde el desarrollo de Internet y, posteriormente, desde la creación de la World Wide Web. Numerosas tareas profesionales y personales se ven simplificadas y agilizadas gracias a su uso. Y el ámbito de la enseñanza es un claro ejemplo de este hecho.

En particular, la enseñanza de lenguas siempre se ha caracterizado por la utilización de cualquier tecnología que mejorara, en el sentido de que fuera, el proceso de adquisición de una lengua. Así hemos utilizado libros, diccionarios, periódicos, pizarras, casetes, proyectores, televisión, vídeo... y al poco tiempo de aparecer los ordenadores se incorporaron en las clases de lengua.

\section{El aprendizaje de lenguas asistido por ordenador (ALAO)}

La disciplina que se ocupa de este campo de investigación es conocida por la sigla CALL, Computer Assisted Language Learning o el equivalente en nuestro contexto ALAO, Aprendizaje de lenguas asistido por ordenador. Estas siglas conviven con otras que señalan alguna vertiente de las múltiples que tiene este ámbito científico. Así encontramos NBLT, Network-Based Language Teaching o Enseñanza de lenguas basada en la web, WELL, WebEnhanced Language Learning o Aprendizaje de idiomas mejorado en la web , ICALL, Intelligent Computer Assisted Language Learning o Aprendizaje de lenguas asistido por computadora inteligente, MALL, Mobile Assisted Language Learning o Aprendizaje de lenguas asisitido por dispositivos móviles, etc.

En esta investigación utilizamos la sigla ALAO por considerarla la más genérica y porque da cabida a todas y cada una de las múltiples modalidades que se basan o usan la informática para el aprendizaje de lenguas.

El ALAO se inició durante los años 60 del siglo pasado, con los primeros ordenadores, pero no fue hasta los años 80 cuando se extendió. Aunque en la década siguiente, en los 90 , con la aparición del CD-ROM fue cuando se produjo una verdadera expansión. Posteriormente, el surgimiento de Internet, la web y los avances tecnológicos constantes han superado muchas de las barreras y propulsado el aprendizaje lingüístico como no había sido imaginado antes. Por su parte, el estudio ALAO alcanza tanto los aspectos de análisis previos para el desarrollo de materiales, como la creación, la implementación y la evaluación de los resultados obtenidos. Es decir, aglutina todos los aspectos susceptibles de estudio del aprendizaje de lenguas asistido por ordenador.

Más recientemente, con los recursos de la web 2.0, junto a los dispositivos móviles, se están ampliando las posibilidades del aprendizaje lingüístico a través de la red. La mayoría de los recursos de ALAO se desarrollan a través de las aplicaciones de la web 2.0 y para utilizarse en plataformas diversas, en contraste con las etapas anteriores en que predominaba el uso del ordenador local. Si uno de los mayores déficits de los materiales para la adquisición de lenguas a través de la tecnología era la imposibilidad de interactuar de forma significativa

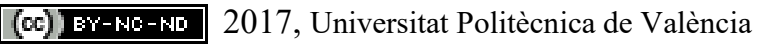


con otro hablante, ahora ha desaparecido. Contamos con numerosas y variadas herramientas de comunicación, de expresión y de colaboración. Se ha flexibilizado la modalidad de acceso y se han diversificado los formatos. Lo cual ha provocado la proliferación de materiales y cursos para el aprendizaje de lenguas.

\section{El aprendizaje de español como lengua extranjera a través de la web}

Actualmente existen numerosísimos recursos informáticos para el auto aprendizaje de español como lengua extranjera (ELE) puesto que la demanda social es cada vez mayor, tanto para recursos web como para dispositivos móviles, que dotan al usuario de libertad y comodidad. Sin embargo, esta oferta no es tan completa como cabría esperar, como veremos, la mayor parte de los recursos web que existen actualmente no tratan la complejidad que requiere la adquisición de la competencia comunicativa en su totalidad (Cruz, 2002, 2014).

Hemos analizado los 50 portales web para el aprendizaje del ELE mejor posicionados en Google para comprobar qué tipo de aprendizaje proporcionan. Así, hemos podido constatar que los recursos pertenecientes a instituciones públicas conforman solo tres del total analizado, estos son: AVE, Radio Fle y Aprender ELE-UPV. En su totalidad son recursos que denotan un trabajo muy cuidado de la enseñanza de español, aunque no llegan a trabajar la competencia comunicativa oral, que es la que nos ocupa en esta investigación. El aprendiz puede escuchar ejemplos de uso de la lengua, como por ejemplo en Radio FLE, pero no puede llegar a interaccionar con otros usuarios.

En segundo lugar, de los recursos analizados, el 19\% son gestionados por docentes de ELE. En este grupo sobresalen algunos como Blog para aprender español, A vueltas con E/LE o Charla en español, entre otros. Se observa que se centran en los problemas que suelen tener los aprendices de español focalizándose en temas gramaticales como la formación verbal.

En tercer lugar, los sitios web analizados pertenecen mayoritariamente a empresas privadas, en este grupo encontramos dos subtipos, por un lado, pequeñas empresas o academias de idiomas que formalizan una web que complementa la enseñanza en el aula como Lingolia. Y por otro, grandes empresas privadas que crean recursos web y para dispositivos móviles para el auto aprendizaje de idiomas y no solo español, este es el caso de Duolingo, Rosetta Stone o Fluencia. Estos recursos comienzan a trabajar la competencia comunicativa oral mediante la repetición de frases o palabras, no obstante, no podemos entender que este trabajo sea completo, puesto que el estudiante aprende a pronunciar palabras y tonos. No debemos olvidar que la lengua es un instrumento de comunicación (Albelda \& Fernández, 2006) y el fin del aprendizaje de una lengua es adquirir la capacidad de comunicarse usándola.

En conjunto, los recursos analizados trabajan la competencia comunicativa escrita pero limitan la práctica de la lengua oral a la repetición y la pronunciación de frases. 


\section{Desarrollo de la innovación}

Como hemos podido comprobar en los apartados anteriores, los recursos web de auto aprendizaje en la web están centrados en aspectos gramaticales y estructuralistas, en el aprendizaje por repetición de estructuras sintácticas en el plano comunicativo de la lengua. De esta manera, y en base a este análisis, hemos decicido crear un recurso de auto aprendizaje de español como lengua extranjera con las aportaciones tecnológicas del procesamiento del habla (PLN) y aspectos concretos tecnológicos que se necesitan para un recurso de estas características.

$\mathrm{R} / \mathrm{ele} / \mathrm{CCO}$ es un recurso de auto aprendizaje de español como lengua extranjera que tiene como fundamento el educativo los aspectos clave de una conversación en español, y los rasgos que la caracterizan culturalmente y que hacen que su aprendizaje sea complicado. Este es un recurso innovador ya que fomenta el aprendizaje de español con los aspectos más modernos de la ciencias del habla en este idioma mejorando la competencia comunicativa a estudiantes que no tienen a su alcence personas con quien mejorar la competencia comunicativa. Debemos entender que la competencia conversacional es un aspecto fundamental en el aprendizaje de cualquier idioma, no solo en español, por lo que es necesario cada vez con más frecuencia crear herramientas que mejoren estos aspectos. Existen herramientas como Duolingo o Rosetta Stone (entre otras) que comienzan a utilizar las ciencias del habla para el aprendizaje de idiomas mediante ejercicios con base estructuralista. La repetición de frases o sintagmas no es un varemo de mejora de la competencia comunicativa oral ni de la competencia conversacional. Por este motivo, R/ele/CCO es una herramienta de innovación, parte de conversaciones cortas que se van complicando a medida que el alumno completa una unidad didáctica. Y así, partimos del planteamiento de que el estudiante es capaz de mejorar los aspectos intrínsecos de la competencia conversacional mejorando tanto en aspectos de oralidad como gramaticales o de vocabulario. Es decir, el plantemiento que subyace de $\mathrm{R} / \mathrm{ele} / \mathrm{CCO}$ es que mediante la conversación en ese idioma el alumno mejora otros aspectos de la lengua que están plenamente relacionados con la comunicación.

Nuestro recurso actualmente cuenta con los niveles A1 y A2 del Marco Común Europeo de Referencias para las Lenguas, cada nivel está dividido en diferentes unidades didácticas en la que el alumno a parte de conversar con el programa, aprende vocabulario y estructuras sintácticas y gramaticales, todo ello, sin olvidar el plano comunicativo, ya que lo hace directamente, conversando.

La pregunta que surge es, si es posible realizar este tipo de recurso por qué no existen en el plano actual. Para ello debemos de entender que, el PLN es un ciencia tecnológica en constante evolución, hay aspectos que aún están en desarrollo, sobre todo, en relación con el español, lengua menos desarrollada que otras como el inglés. En un primer momento, creamos R/ele/CCO siguiendo las directrices de Sphinx ${ }^{1}$ mediante el lenguaje informático

${ }^{1}$ Tutorial creado por los expertos de la Universidad de Carnegie Mellon (Pensilvania)

(c) EY-NC-ND 2017, Universitat Politècnica de València 
Java. Entorno a esto, nos encontramos con problemas, el principal, que el ordenador no era capaz de entender los sonidos de personas no experimentadas con el idioma, es decir, nuestro futuros estudiantes. El programa acudia al corpus intrínseco en él y limitado, y no encontraba las coincidencias necesarias. Llisterri (2003: 6) evalúa los problemas en las pruebas de sistemas de conversión de texto en habla y viceversa en tres parámetros como la inteligibilidad, la comprensión y la calidad global que se ve mermada por los dos anteriores. Los dos primeros aspectos se veían marcados en nuestras primeras pruebas con el recurso.

Por ello, comenzamos a navegar en la red y encontramos un procesador de habla a texto con mayor corpus y menos tiempo de respuesta, dicho procesador es IBMWatson ${ }^{2}$. Watson supone una mejora para nuestro recurso, porque no cuenta con un corpus, es decir, es un software inteligente, que aprende con cada pregunta y respuesta, por esto, es más comodo trabajar con Watson que con el medio anterior. Watson nos permite trabajar el procesamiento del lenguaje natural y el procesamiento de habla a texto desde una perspectiva más inteligente, aprendiendo cada día más.

Pues bien, ahora connocemos todo lo que puede hacer la informática por el aprendizaje de segundas lenguas, hemos de atender a aspectos pedagógicos necesarios para la creación de un recurso web de auto aprendizaje de segundas lenguas. Para la comodidad de estudiante, necesitamos las siguientes características:

1. La creación de una vía de comunicación bidireccional entre la web y el usuario, es decir, un apartado centrado para que el aprendiz pueda consultar dudas de cualquier tipo con el recurso con la mayor facilidad.

2. El registro del trabajo del alumno en el recurso es fundamental, mediante un registro inicial el estudiante podrá acceder a su trabajo en el mismo punto en el que se quedó en la ocasión anterior.

3. En cuanto a los contenido, pensamos que nuestro recurso debe fundamentalmente, presentar una estructuración, secuenciación, flexibilidad y presentación de los contenidos a trabajar. Es primordial, dotar al alumno de las pautas necesarias para elegir, en la medida de lo posible, los puntos que quiere estudiar.

4. R/ele/CCO está pensado para responder al alumno con una retroalimentación formativa, intentando dar al aprendiz la solución a su error, haciendo del error algo en lo que aprender.

5. Otro de los aspectos fundamentales de $\mathrm{R} / \mathrm{ele} / \mathrm{CCO}$ es, la base de las conversaciones auténticas y naturales. Los ejercicios de nuestro recurso son las conversaciones del estudiante con el ordenador, estas conversaciones son lo más real posible, desde presentaciones hasta conversaciones que van complicándose en base a la dificultad adquirida en torno a los niveles del MCERL. Con este aspecto, ligamos el aspecto de conciencia lingüística, necesario para un correcto auto aprendizaje de la lengua.

$\mathrm{R} / \mathrm{ele} / \mathrm{CCO}$ tiene una estrucutra joven que intenta transmitir un ambiente de confianza en el proceso de auto aprendizaje de español. Para ello, hemos utilizado imágenes libres de

${ }^{2}$ http://www-03.ibm.com/marketing/mx/watson/watson-for-a-smarter-planet/index.html 
derechos de autor que focalizan desde el primer momento el proceso de auto aprendizaje de la conversación en el recurso.

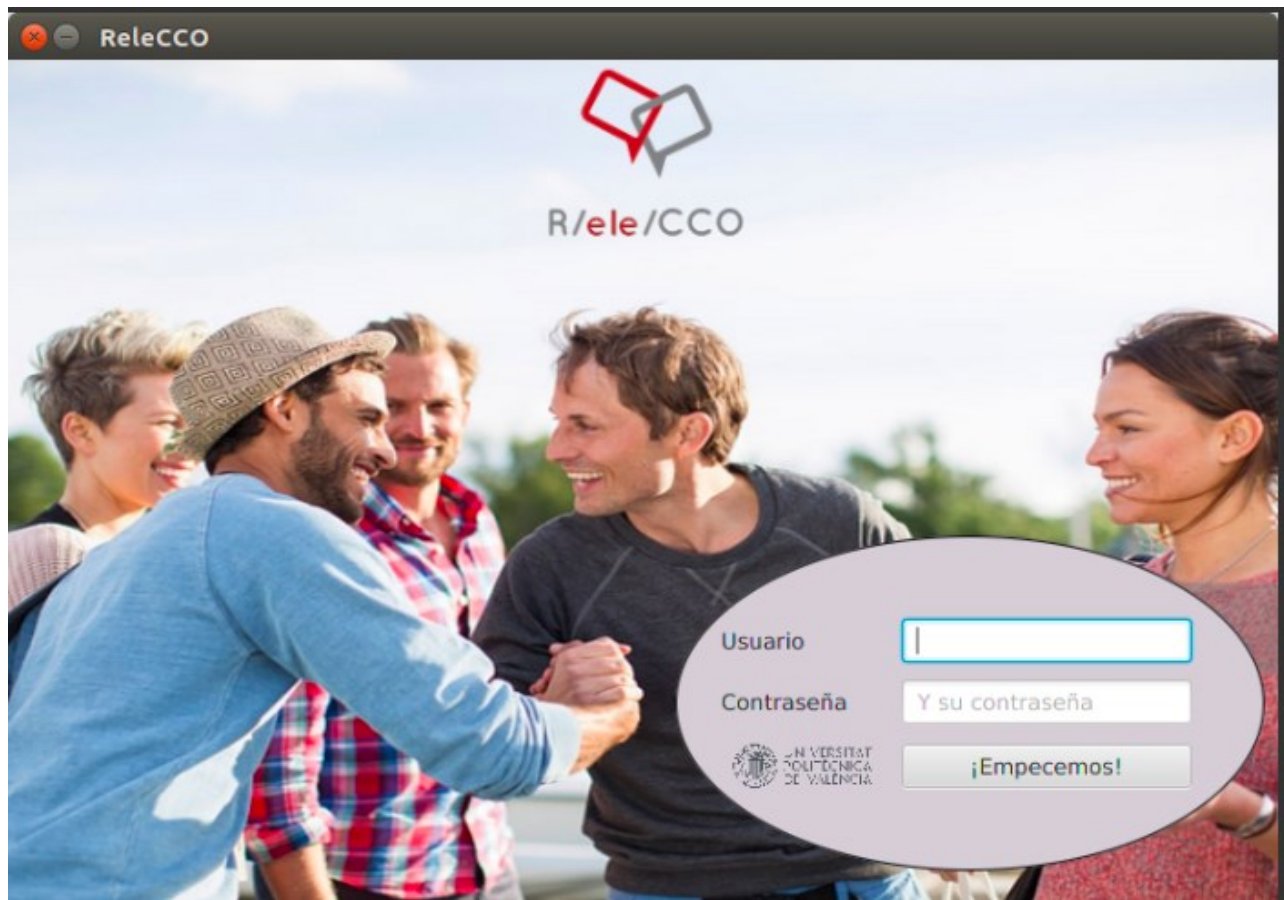

Imagen 1: R/ele/CCO

En pantallas posteriores el alumno tiene en su alcence diferentes aspectos del programa por los que navegar como: características, contacto y niveles de aprendizaje con los temas a tratar en cada unidad, dando la libertad al estudiante de elegir el tema que quiera trabajar. Pensamos que es necesario dotar al estudiante de libertad de elección sin crear una ruta prediseñada de aprendizaje.

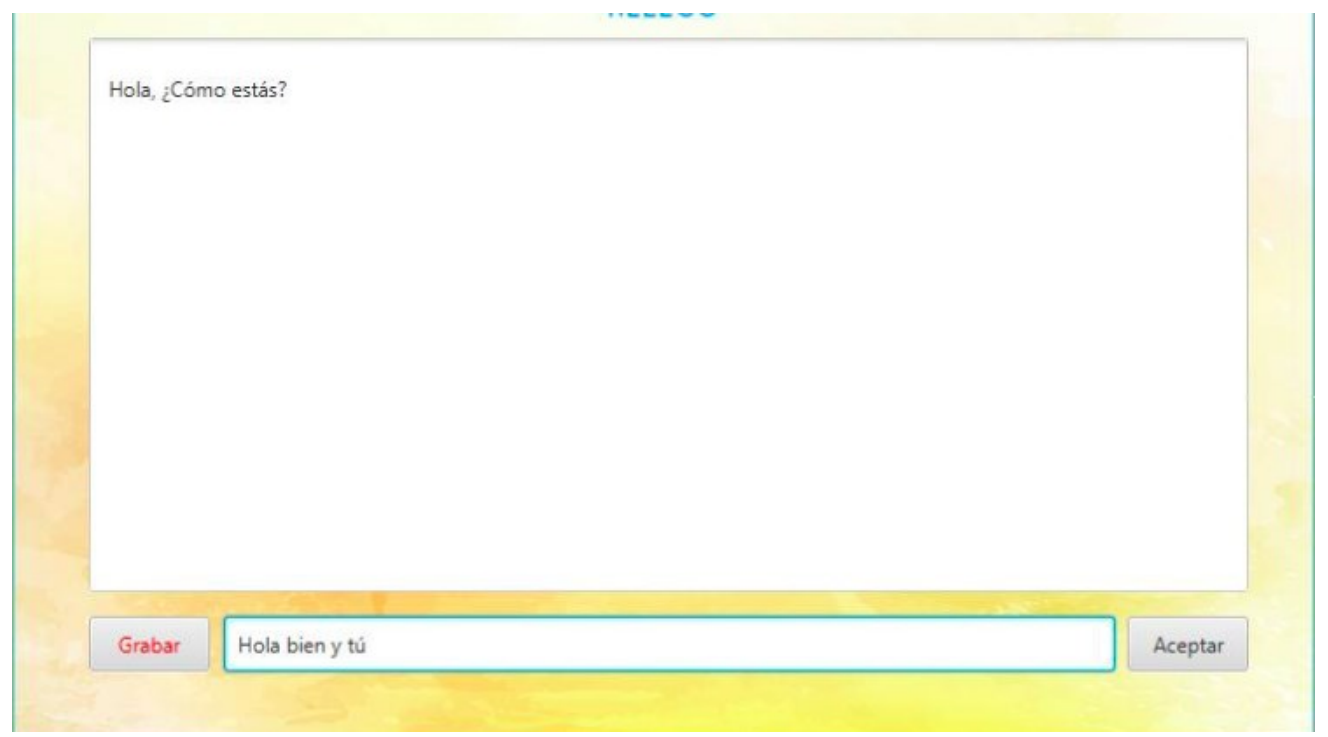

(cc) EY-NC-ND 2017, Universitat Politècnica de València 
Imagen 2: R/ele/CCO

Como podemos observar en esta pantalla, la conversación entre ordenador y estudiante funciona de la siguiente manera: el ordenador es el primero en comenzar con el turno de habla y el estudiante (con un micrófono) efecturá el segundo turno de habla y cuando esté conforme con lo que ha dicho enviará el mensaje, creando así una conversación en tiempo real con la que mejorar rasgos comunicativos en español.

No obstante, no podemos ofrecer resultados sobre la efectividad y la opinión de los usuarios porque estamos a la espera de sacar el recurso tanto en versión web como móvil.

Para concluir, pensamos que esta web es capaz de cubrir las necesidades de los estudiantes de español como lengua extranjera para con la competencia comunicativa oral en dicha lengua, utilizando las innovaciones tecnológicas necesarias para llevar a cabo el proceso de auto aprendizaje del español como segunda lengua.

\section{Conclusiones}

A lo largo de este artículo, hemos visto que tanto las tecnología del habla como las tecnologías de la comunicación han fundamentado los cambios necesarios para el auto aprendizaje de lenguas asisitido por ordenador y actualmente, también por dispositivos móviles. Asimismo, hemos visto que los recursos de auto aprendizaje de español carecen de las herramientas necesarias para el trabajo de la competencia comunicativa oral, fundamentando la enseñanza en otros aspectos y vinculando esta competencia a la repetición de estructuras sintácticas.

Por eso, $\mathrm{R} /$ ele/CCO supone un cambio en el concepto de auto aprendizaje de la competencia comunicativa oral en contrapunto a los recursos de auto aprendizaje de español como lengua extranjera. Atendiendo al panorama actual, comprobamos que es necesario que un recurso web se fundamente en torno a las novedades y ventajas que la tecnología nos brinda para poder hacer realidad las necesidades de aprendizaje del estudiante de español como lengua extranjera.

\section{Referencias}

ALBELDA, M., \& FERNÁNDEZ, M.J. (2006). La enseñanza de los registros lingüísticos en E/LE. Una aplicación a la conversación coloquial. Revista de didáctica: MarcoELE, 3, 1-31

CRUZ, M. (2002). Enseñar español en la era de Internet. Las www y la enseñanza del español como lengua extranjera. Barcelona, España: Editorial Octaédro.

CRUZ, M. (2014). Veinte años de tecnologías y ELE. Reflexiones en torno a la enseñanza de español como lengua extranjera en la era de Internet. Revista de didáctica: Marco ELE, 19, 1-18.

IBMWATSON. Watson para un mundo más inteligente. <http://www03.ibm.com/marketing/mx/watson/watson-for-a-smarter-planet/index.html> [Consulta:2 de abril de 2016] 
Llisterri, J. (2003). "Las tecnología del habla : entre la ingeniería y la lingüística" En Actas del I Congreso Internacional La ciencia ante el públio. Cultura humanística y desarrollo tecnológico. Salamanca : Instituto Universitario de Estudios de la Ciencia y la Tecnología.

MCERL (2002). Marco Común Europeo de Referencia de Lenguas. Madrid, España: Instituto Cervantes. 